ERRATUM

\title{
Erratum to: On the length of arithmetic progressions in linear combinations of $S$-units
}

\author{
LAJOS HAJdu AND FLORIAN LUCA
}

\section{Erratum to: Arch. Math. (2010) 94:357-363 DOI 10.1007/s00013-010-0111-7}

In formulating Lemma 2.1 in the original paper, a result of Amoroso and Viada [1] is interpreted in a wrong way. We are grateful to Kálmán Győry for calling our attention to this error. The correct formulation of the lemma is the following.

Lemma 2.1. Equation (2.1) has at most $C(k, r):=(8 k)^{4 k^{4}\left(k+r^{k}+1\right)}$ nondegenerate solutions $\left(x_{1}, \ldots, x_{k}\right) \in \Gamma^{k}$.

Proof. Since the rank of $\Gamma^{k}$ is at most $r^{k}$, this is an immediate consequence of Theorem 6.2 in [1], indeed.

Unfortunately, this error affects the bound in Theorem 1.1 in the original paper. Now we give a correct formulation of this result and indicate the main changes in the proof (which are only technical ones).

Theorem 1.1. We have

$$
L<\exp \left((8(n+t+r))^{8(2 r+2)^{2 t+2}}\right) .
$$

Proof. The change in Lemma 2.1 implies simple automatic changes in the proof. These lead to the correct form of (2.4), given by

$$
|\mathcal{I}|<n^{t} 2^{t+1}(8(t+1))^{4(t+1)^{4}\left(t+(r+s)^{t+1}+2\right)} .
$$

Then, at the end of the proof making the choice $s=r+2$, the statement follows by a simple calculation.

The online version of the original article can be found under doi:10.1007/s00013-010-0111-7. 


\section{Reference}

[1] F. Amoroso and E. Viada, Small points on subvarieties of a torus, Duke Math. J. 150 (2009), 407-442.

LAJOS HAJdu

Number Theory Research Group of the Hungarian Academy of Sciences, Institute of Mathematics, University of Debrecen, P.O. Box 12, 4010 Debrecen, Hungary

e-mail: hajdul@math.klte.hu

FLORIAN LUCA

Mathematical Institute, UNAM, Ap. Postal 61-3 (Xangari), CP 58089 Morelia, Michoacán, Mexico

e-mail: fluca@matmor.unam.mx 